\title{
Rotationally inelastic molecular scattering. Computational tests of some simple solutions of the strong coupling problem
}

\author{
Thomas P. Tsien \\ Gregory A. Parker \\ parker@nhn.ou.edu \\ Russell T. Pack
}

Follow this and additional works at: https://scholarsarchive.byu.edu/facpub

Part of the Biochemistry Commons, and the Chemistry Commons

\section{Original Publication Citation}

Tsien, Thomas P., Gregory A. Parker, and Russell T. Pack."Rotationally inelastic molecular scattering. Computational tests of some simple solutions of the strong coupling problem." The Journal of Chemical Physics 59 (1973): 5373-5381.

\section{BYU ScholarsArchive Citation}

Tsien, Thomas P.; Parker, Gregory A.; and Pack, Russell T., "Rotationally inelastic molecular scattering. Computational tests of some simple solutions of the strong coupling problem" (1973). Faculty Publications. 785.

https://scholarsarchive.byu.edu/facpub/785 


\title{
Rotationally inelastic molecular scattering. Computational tests of some simple solutions of the strong coupling problem*
}

\author{
Thomas P. Tsien $\dagger$, Gregory A. Parker, and Russell T Pack $\ddagger$ \\ Department of Chemistry, Brigham Young University, Provo, Utah 84602
}

(Received 13 August 1973)

\begin{abstract}
Partial cross sections (opacity functions) for rotational transitions in atom-diatom collisions are computed in the infinite-order sudden (IOS) approximation and compared with accurate close-coupling (CC) calculations. Agreement is good in the dominant coupling (small total angular momentum $J$ ) region. Simple methods for calculating integral inelastic cross sections are discussed, and it is found that accurate cross sections can often be computed very simply, even when large numbers of channels are coupled together, by using IOS or first-order sudden (FOS) approximations for small $J$ and CC or exponential Born (EBDW) methods for large $J$.
\end{abstract}

\section{INTRODUCTION}

A major problem in the quantum theory of molecular scattering is that (for small total angular momentum $J$ and any molecules except hydrides) the collision causes large numbers of rotational states to be strongly coupled together. This gives rise to a large set of coupled radial Schrödinger equations which must be solved simultaneously. Although truly significant advances ${ }^{1,2}$ have been made in recent years in the accurate, close-coupled (CC) numerical solution of these equations, the $\mathrm{CC}$ computation of the large numbers of cross sections needed in the description of relaxation and reactive processes is still exceedingly expensive. Hence, there remains a real need for approximate methods which give accurate results and yet are computationally simple.

Most simple approximations (such as the ordinary distorted wave approximation) fail miserably in the dominant coupling (small $J$ ) region. Besides the sudden approximations used herein, the only other computationally simple method that we know of that may be useful here is the statistical approximation. ${ }^{3}$ But it cannot be used unless one knows from some other source how many channels are strongly coupled together, ${ }^{4}$ and it does not give the phase oscillations observed in the figures presented in Sec. III. Hence, it was not used in this work.

In this paper we report calculations comparing some sudden approximations with accurate $\mathrm{CC}$ results for several atom-diatom rotationally inelastic collisons using empirical intermolecular potentials. In Sec. II the problem and methods used are outlined; then, in Sec. III, results are presented and comparisons made. We conclude in Sec. IV that simple but adequate approximate solutions of the rotationally inelastic scattering problem are now known.

\section{THEORY AND CALCULATIONS}

Since the approximations used in this paper have all been discussed elsewhere, only a sketch of their derivations is given.

The objective here is to solve the Schrödinger equation for the collision of an atom $\mathrm{A}$ with a diatomic molecule BC. To get at the essential difficulty of treating rotational states, we use the rigid rotor model and formulation of Arthurs and Dalgarno ${ }^{5}$ with empirical intermolecular potentials $U$ of the form

$$
U\left(r^{\prime}, \theta\right)=V\left(r^{\prime}\right)+W\left(r^{\prime}\right) P_{2}(\cos \theta),
$$

where the coordinates are those shown in Fig. 1 and the primes stand for cgs units. In reduced units $\left(r=r^{\prime} / \sigma\right.$, where $\sigma$ is the point at which $V$ is zero), the coupled equations which must be solved can be written in the form ${ }^{5}$

$$
\left[1 d^{2} / d r^{2}+\mathbf{E}-V(r) 1-W(r) \mathbf{F}\right] \mathbf{G}(r)=0,
$$

where $\mathbf{G}=\left\{G_{n}(r)\right\}$ is a column vector of radial channel wavefunctions, $F$ is a matrix of Percival-Seaton $^{3,5,6}$ coefficients, 1 is the unit matrix, and the elements of $\mathbf{E}$ are

$$
E_{m n n}=\delta_{m n}\left[k_{n}^{2}-l_{n}\left(l_{n}+1\right) / r^{2}\right],
$$

where the $l_{n}$ are relative orbital angular momenta. In these reduced units the channel wavenumbers $k_{n}$ and energies $k_{n}^{2}$ are given by

$$
k_{n}^{2}=2 \mu \sigma^{2} E / \hbar^{2}-B j_{n}\left(j_{n}+1\right),
$$

where $\mu$ is the atom-diatom reduced mass, $E$ is the total energy (in cgs units), and the $j_{n}$ are the rotor angular momentum quantum numbers. The rotational constant $B$ of the diatomic molecule is given in these units by

$$
B=\mu \sigma^{2} / I_{\mathrm{BC}}=\mu_{A-B C} \sigma^{2} / \mu_{\mathrm{BC}} R^{\prime 2}
$$

where $R^{\prime}$ is the average BC internuclear distance, 


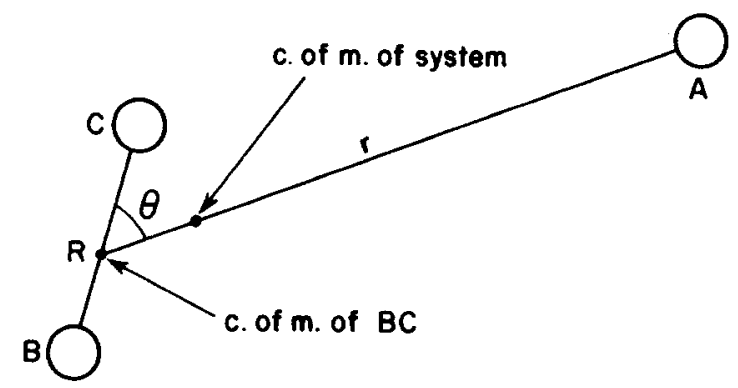

FIG. 1. Center of mass coordinates used herein for A $+\mathrm{BC}$ collisions.

and $\mu_{\mathrm{BC}}$ is the reduced mass of $\mathrm{BC}$. The potentials used herein are (in reduced units)

$$
V=4 D\left(r^{-12}-r^{-6}\right),
$$

and

$$
W=4 D\left(a_{12} r^{-12}-a_{6} r^{-6}\right)
$$

where $D=2 \mu_{\mathrm{A}-\mathrm{BC}} \sigma^{2} \epsilon / \hbar^{2}$. Here $\epsilon$ is the (cgs) depth of the intermolecular potential and $a_{12}$ and $a_{6}$ are asymmetry parameters.

For each value of the total angular momentum $J$, one must solve the set of coupled equations (2) subject to the boundary conditions

$$
\begin{gathered}
G_{n} \underset{r \rightarrow \infty}{\sim} k_{n}^{-1 / 2}\left\{\delta_{n i} \exp \left[-i\left(k_{i} r-\frac{1}{2} l_{i} \pi\right)\right]\right. \\
\left.-S_{n i}^{J} \exp \left[i\left(k_{n} r-\frac{1}{2} l_{n} \pi\right)\right]\right\}
\end{gathered}
$$

to obtain the elements $S_{n i}^{J}$ of the scattering matrix. Once this is done the degeneracy-averaged integral cross sections needed can be written in the form ${ }^{5}$

$$
\sigma\left(j_{m}-j_{n}\right)=\left(\pi / k_{n}^{2}\right) \sum_{J=0}^{\infty}(2 J+1) \Theta_{J}\left(j_{m}, j_{n}\right),
$$

where the opacity functions (average transition probabilities ) $\rho_{J}\left(j_{m}, j_{n}\right)$ are given by ${ }^{7}$

$\odot_{J}\left(j_{m}, j_{n}\right)=\left(2 j_{n}+1\right)^{-1} \sum_{\xi_{n}=\left|J-j_{n}\right|}^{J+f_{n}} \sum_{l_{m}=\left|J-j_{m}\right|}^{J+j_{m}}\left|\delta_{m n}-S_{m n}^{J}\right|^{2}$.

To compute "exact" cross sections, one truncates the infinite set of coupled equations by restricting the number of rotational states $j_{n}$ included, solves the resulting finite set of equations numerically using some CC method and then adds additional rotor states $j_{n}$ (and their associated $l_{n}$ ) and repeats the $\mathrm{CC}$ calculations until the desired opacity functions converge to within some specified accuracy. The CC computations reported here were performed using Gordon's method ${ }^{1}$ and program. ${ }^{8}$ The results are accurate but expensive.

Many simple approximate solutions of (2), such as the ordinary distorted-wave (DW) methods, often give ridiculous results for the systems considered here. For example, the DW $\sigma(2-0)$ for the Ar + TlF example in Sec. III is over ten times too large, and the DW $\sigma(4-0)$ is identically zero. At the very least, one must have a many-state, probability-conserving approximation. One such approximation which we have previously discuss ed $d^{9,10}$ is the strong coupling or infinite-order sudden (IOS) approximation, appropriate for the small $J$ regions where the elements of WF dominate the differences in the elements of $\mathrm{E}$ and strongly couple the equations together. In the IOS approximation one sets $k_{n}=k$ and $l_{n}=l$ for all the strongly coupled channels. Then, the resulting equations can be solved exactly: Let $G=U g$, where $\mathrm{U}$ is the $r$-independent unitary transformation which makes $\mathrm{U}^{\dagger} \mathrm{FU}=\Lambda$ diagonal. Then, the elements of $\mathbf{g}$ satisfy uncoupled equations and the resulting $S$ matrix is ${ }^{9,10}$

$$
\mathbf{S}^{J}=\mathbf{U B U}^{\dagger}=\exp \left(2 i \mathbf{U} \eta \mathbf{U}^{\dagger}\right) \text {, }
$$

where

$$
B_{m n}=\delta_{m n} \exp \left(2 i \eta_{n}^{s c}\right), \quad .
$$

and

$$
(\eta)_{m n}=\delta_{m \eta} \eta_{n}^{s c}
$$

The WKB approximation for the phase shifts $\eta_{n}^{\text {sc }}$ is

$$
\begin{aligned}
\eta_{n}^{s c}= & \int_{r_{n}}^{\infty}\left\{\left[k^{2}-\left(l+\frac{1}{2}\right)^{2} / r^{2}-V-W \Lambda_{n}\right]^{1 / 2}-k\right\} d r \\
& -k r_{n}+\frac{1}{2}\left(l+\frac{1}{2}\right) \pi,
\end{aligned}
$$

where $r_{n}$ is the turning point and $\Lambda_{n}$ an element of the diagonal matrix $\Lambda$. Thus, an $N$ state IOS calculation at a given $J$ simply requires the diagonalization of one $N \times N$ matrix $\mathbf{F}$ and evaluation of $N$ WKB phase shifts. In the IOS computations reported here, the Jacobi method was used for the diagonalization and a Simpson's rule numerical integration with increasing step size was used for the phase shifts.

As we have noted previously, ${ }^{10}$ if one expands (14) in powers of $\Lambda_{n}$ and keeps only the terms through $\mathcal{O}\left(\Lambda_{n}\right)$, the resulting scattering matrix,

$$
\mathbf{S}^{J}=\exp \left[2 i\left(\eta^{(0)} 1+\eta^{(1)} \mathbf{F}\right)\right]
$$

is precisely that of the familiar semiclassical first-order sudden (FOS) approximation. ${ }^{11}$ Here

$$
\begin{aligned}
\eta^{(0)}= & \int_{r_{0}}^{\infty}\left\{\left[k^{2}-\left(l+\frac{1}{2}\right)^{2} / r^{2}-V\right]^{1 / 2}-k\right\} d r \\
& -k r_{0}+\frac{1}{2}\left(l+\frac{1}{2}\right) \pi,
\end{aligned}
$$

and

$$
\eta^{(1)}=-\frac{1}{2} \int_{r_{0}}^{\infty} \frac{W(r)}{\left[k^{2}-\left(l+\frac{1}{2}\right)^{2} / r^{2}-V\right]^{1 / 2}} d r .
$$

Thus, $N$ state FOS calculations require evaluation of just two phase integrals and expansion of the 
TABLE I. Parameters used herein. $\mu_{\mathrm{A}-\mathrm{BC}}$ is the reduced mass of $\mathrm{A}+\mathrm{BC}$ in atomic mass units; $\sigma$ is the size parameter of the 12-6 potential in angstroms; $a_{12}$ and $a_{6}$ are the repulsive and attractive asymmetry parameters, respectively; $\epsilon / k_{B}$ is the depth of the potential well in ${ }^{\circ} \mathrm{K} ; B^{\prime}$ is the rotational constant of the diatomic molecule in $\mathrm{cm}^{-1} ; E / k_{B}$ is the total relative energy available in ${ }^{\circ} \mathrm{K} ; D$ is the reduced well depth; $B$ the reduced rotational constant; and $k^{2}(j=0)$ the reduced total energy.

\begin{tabular}{lccccccccccc}
\hline System & $\mu_{\mathrm{A}-\mathrm{BC}}(\mathrm{amu})$ & $\sigma(\AA)$ & $a_{12}$ & \multicolumn{1}{c}{$a_{6}$} & $\epsilon / k_{B}\left({ }^{\circ} \mathrm{K}\right)$ & $B^{\prime}\left(\mathrm{cm}^{-1}\right)$ & $E / k_{B}\left({ }^{\circ} \mathrm{K}\right)$ & $D$ & $B$ & $k^{2}(j=0)$ & $R e f s$. \\
\hline $\mathrm{Ar}-\mathrm{TlF}$ & 33.89 & 4.62 & 0.50 & 0.30 & 132.5 & 0.22246 & 1344 & 3947.8 & 9.541 & 40,071 & $13, \mathrm{a}$ \\
$\mathrm{Ar}-\mathrm{N}_{2}$ & 16.47 & 3.50 & 0.50 & 0.13 & 119.5 & 2.010 & 300 & 994.2 & 24.06 & 2495.9 & 16 \\
$\mathrm{Ar}-\mathrm{F}_{2}$ & 19.48 & 3.550 & 0.200 & 0.200 & 115.9 & 0.862 & 300 & $\mathbf{1 1 7 3 .}$ & $\mathbf{1 2 . 5 5}$ & 3036 & $18, \mathrm{~b}$ \\
$\mathrm{Ar}-\mathrm{Cl}_{2}$ & 25.55 & 3.831 & 0.200 & 0.200 & 190.7 & 0.2438 & 300 & 2948. & 5.423 & 4638 & $18, \mathrm{~b}$ \\
$\mathrm{Ar}-\mathrm{Br}_{2}$ & 31.96 & 3.837 & 0.200 & 0.200 & 249.8 & 0.08091 & 300 & 4846. & 2.258 & 5820 & $18, b$ \\
$\mathrm{Ar}-\mathrm{I}_{2}$ & 34.52 & 4.146 & 0.200 & 0.200 & 256.9 & 0.03736 & 300 & 6285. & 1.315 & 7339 & $18, \mathrm{~b}$ \\
\hline
\end{tabular}

${ }^{2}$ R. K. Ritchie and H. Lew, Can. J. Phys. 43, 1701 (1965).

${ }^{b}$. Herzberg, Spectra of Diatomic Molecules (Van Nostrand Reinhold, New York, 1950), 2nd edition.

exponential of a matrix. In this work direct expansion of (15) was found to require as many as 30 terms for convergence. To decrease this number and avoid roundoff error, a Chebyshev polynomial contraction of the expansion was made.

For the moderate- and weak-coupling region (large $J$ ) the sudden approximations are not accurate. However, the number of channels $(N)$ required in this region is quite small, so that one can often afford to do the CC calculations. Also, one could use the exponential distorted wave approximations (EDW) discussed by Levine. ${ }^{12}$ These start with Eq. (2) and treat WF as the perturbation in an exponential perturbation method to get a unitary $S$ matrix. If one uses the Born approximation as zeroth order, the resulting EBDW cal- culations $^{13}$ are easily performed. If one uses a distorted-wave approximation as zeroth order, the resulting EDWD approximation gives better results but is much more expensive computationally, except in those cases in which one can evaluate the necessary integrals using asymptotic methods. ${ }^{10,14}$

The calculations reported herein were carried out using the Brigham Young University IBM $360 / 50$ and 7030 STRETCH computers. For typical many-state calculations the relative running times of the FOS :IOS: CC programs were $1: 2: 90$.

\section{RESULTS}

In this section numerical results for some model problems are presented.

TABLE II. Convergence of partial cross sections as the number of channels $(N)$ is increased. The numbers are the values of $(2 J+1) P_{J}(2 \leftarrow 0)$ for $\mathrm{Ar}+\mathrm{TlF}$ calculated in the infinite order sudden (IOS) and close-coupling (CC) approximations. $J$ is the total angular momentum and $j_{\max }$ the largest rotor $j$ kept.

\begin{tabular}{|c|c|c|c|c|c|c|c|c|c|}
\hline$j_{\max }$ & 4 & 6 & 8 & 10 & 12 & 14 & 16 & 18 & \\
\hline$J$ & $N 9$ & 16 & 25 & 36 & 49 & 64 & 81 & 100 & Method \\
\hline \multirow{2}{*}{0} & 0.433 & 0.209 & 0.182 & 0.040 & 0.085 & 0.079 & 0.078 & 0.078 & IOS \\
\hline & 0.428 & 0.217 & 0.199 & 0.045 & 0.090 & 0.086 & 0.085 & 0.085 & $\mathrm{CC}$ \\
\hline \multirow{2}{*}{20} & 16.1 & 8.78 & 7.18 & 1.65 & 3.43 & 3.11 & 3.11 & & IOS \\
\hline & 16.0 & 9.11 & 7.49 & 1.81 & 3.55 & & & & $\mathrm{CC}$ \\
\hline \multirow{2}{*}{40} & 22.5 & 17.9 & 12.0 & 2.88 & 5.54 & 5.31 & 5.25 & & IOS \\
\hline & 23.0 & 18.4 & 13.6 & 3.40 & 6.10 & & & & $\mathrm{CC}$ \\
\hline \multirow{2}{*}{80} & 0.28 & 26.4 & 3.37 & 0.89 & 1.60 & 1.42 & 1.42 & & $\operatorname{IOS}$ \\
\hline & 1.13 & 26.6 & 5.30 & 0.91 & 1.91 & & & & $\mathrm{CC}$ \\
\hline \multirow{2}{*}{120} & 102.5 & 51.8 & 26.0 & 34.1 & 33.5 & 33.3 & & & IOS \\
\hline & 99.7 & 48.1 & 23.0 & 31.0 & 30.4 & & & & $\mathrm{CC}$ \\
\hline \multirow{2}{*}{160} & 33.7 & 21.5 & 25.6 & 25.5 & 25.5 & 25.5 & & & IOS \\
\hline & 14.2 & 1.84 & 3.40 & 3.17 & 3.16 & & & & $\mathrm{CC}$ \\
\hline \multirow{2}{*}{200} & 2.09 & 2.10 & 2.10 & 2.10 & & & & & IOS \\
\hline & 125.5 & 123.8 & 123.7 & & & & & & $\mathrm{CC}$ \\
\hline
\end{tabular}




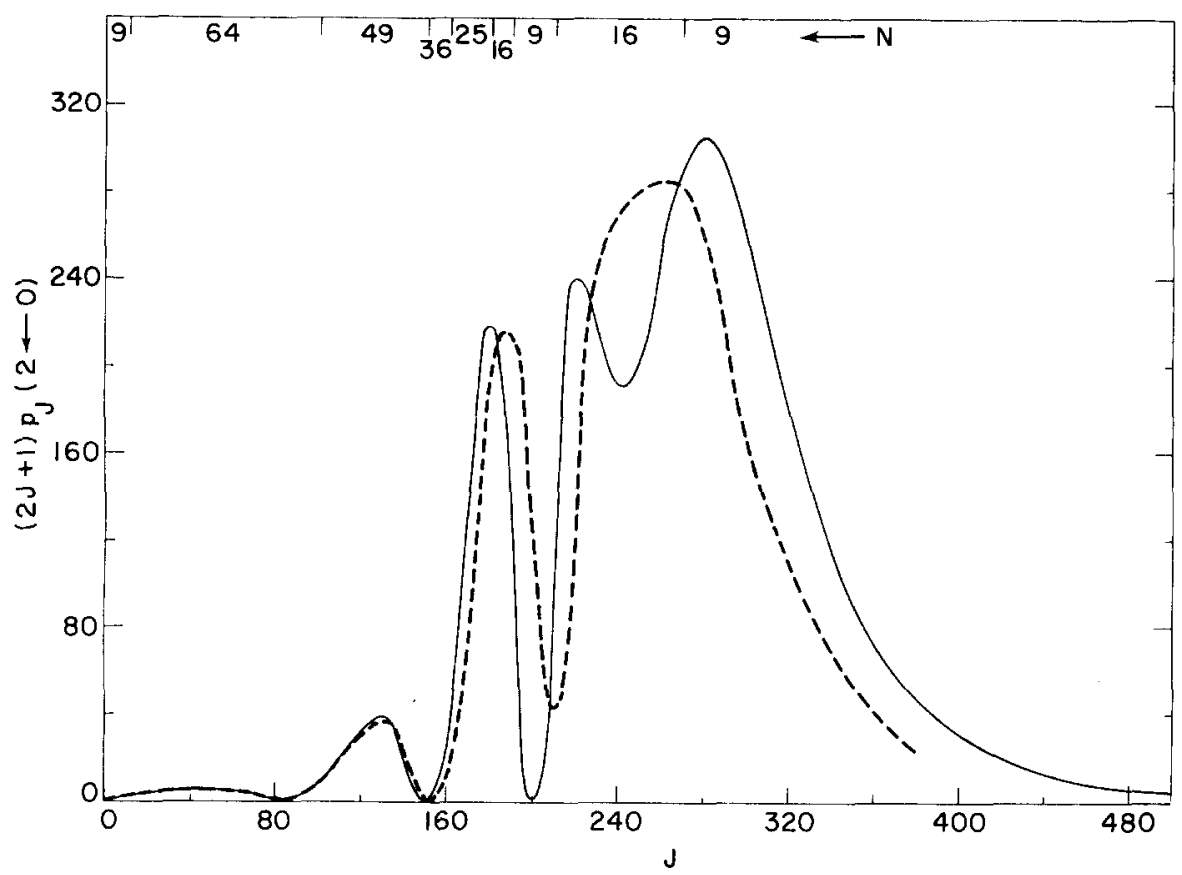

FIG. 2. Behavior of the dimensionless partial cross sections $[(2 J+1)$ times the opacity function $\sigma_{J}$ l as a function of the total angular momentum $J$ for the $j=0$ to $j=2$ transition in an $\mathrm{Ar}-\mathrm{TlF}$ collision. At the top of the figure is the number of channels $N$ required to obtain convergence. Solid line, IOS results; dashed line, $\mathrm{CC}$ results.

\section{A. $\mathrm{Ar}+\mathrm{TIF}$}

The intermolecular potential used in the calculations on $\mathrm{Ar}-\mathrm{TlF}$ rotationally inelastic scattering is that of Balint-Kurti and Levine, ${ }^{13}$ who studied the large $J$ (weak and moderate coupling) opacity functions for this system. This potential (its parameters are listed in Table I) treats TIF as a "homonuclear" diatomic and is thus a crude ${ }^{15}$ model for this particular system, but its behavior is typical of an atom-heavy homonuclear diatom collision.

In this work we calculated cross sections for the $0 \rightarrow 2$ and $0 \rightarrow 4$ rotationally inelastic collisions using the CC, IOS, and FOS approximations. Particular emphasis was placed on determining the dependence of the opacity functions on the number of channels $(N)$ included and on the total angular momentum $J$. The rotational states with $j=0,2, \ldots, j_{\max }$ were

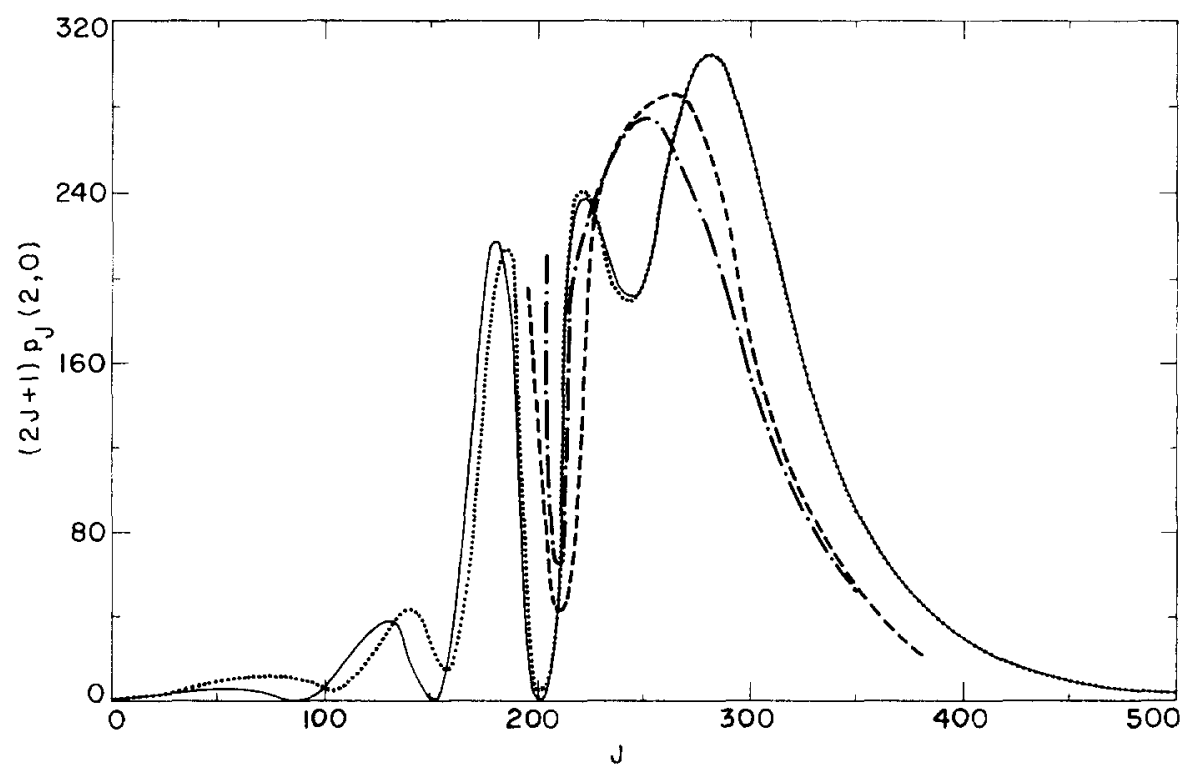

FIG. 3. Comparison of the IOS (solid line), FOS (dotted line), CC (dashed line), and EBDW (dot-dash line) dimensionless partial cross sections $(2 J+1) O_{J}$ $(2 \leftarrow 0)$ for Ar TlF. 


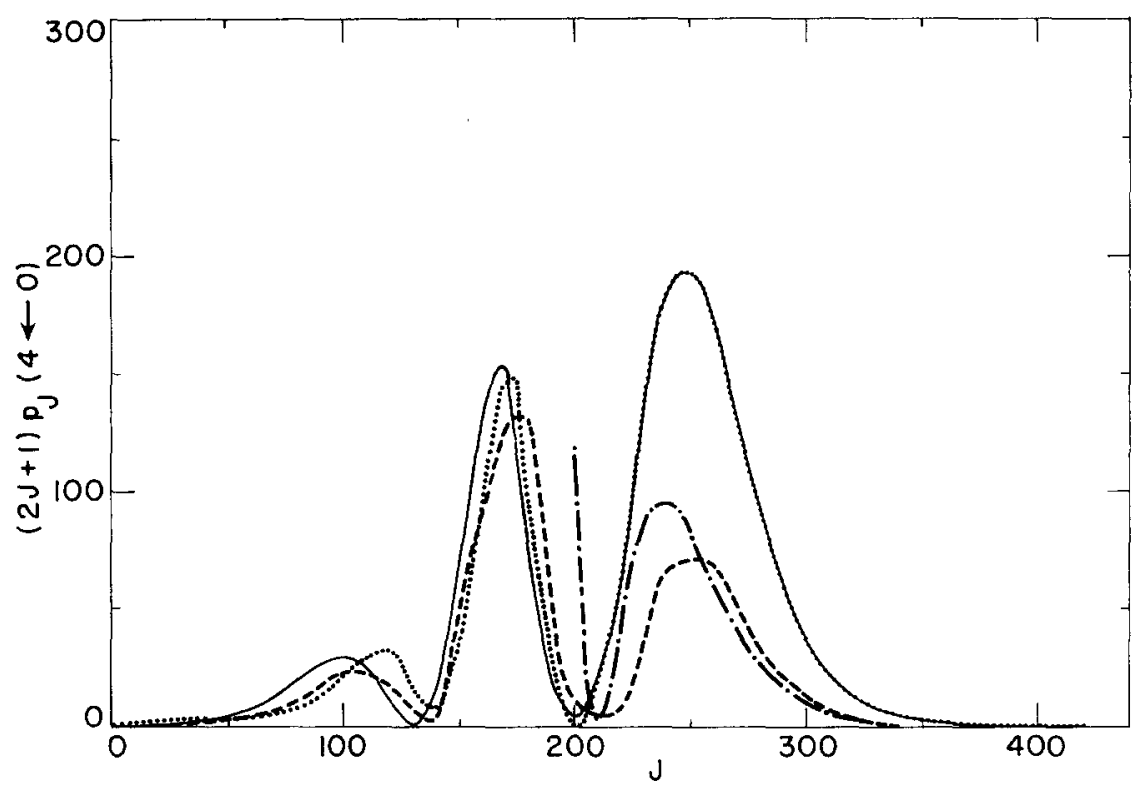

FIG. 4. Dimensionless partial cross sections $(2 J$ +1) $\nabla_{J}(4 \leftarrow 0)$ for $\mathrm{Ar}+\mathrm{TlF}$. The notation is the same as in Fig. 3.

included and $j_{\max }$ was increased until the desired opacity functions converged to a value stable to within about $5 \%$. For a given $j_{\max }$ and $J \geq j_{\max }$, the total number of channels ( $j, l$ combinations) is

$$
N=\sum_{j=0,2, \ldots}^{j_{\max }}(j+1)=\frac{1}{4}\left(j_{\max }+2\right)^{2} .
$$

For very small $J\left(J<j_{\mathrm{max}}\right), N$ is less than this decreasing to $N=\frac{1}{2}\left(j_{\max }+2\right)$ at $J=0$.

The convergence of the opacity functions as $N$ is increased was found to be very similar for the
FOS, IOS, and CC methods and is illustrated by Table II in which the IOS and CC values for $(2 J$ +1) $\mathcal{P}_{J}(2-0)$ are given for a few $J$ values as a function of $N$. One sees immediately that in the small $J(J \lesssim 180$ in this case) region, convergence is neither rapid nor monotonic, and as many as 64 channels are required to obtain satisfactory convergence.

In Fig. 2 the converged $(2 J+1) \rho_{J}(2-0)$ obtained from the IOS and CC methods are plotted vs $J$ for all $J$ to allow easy comparison. At the top of the

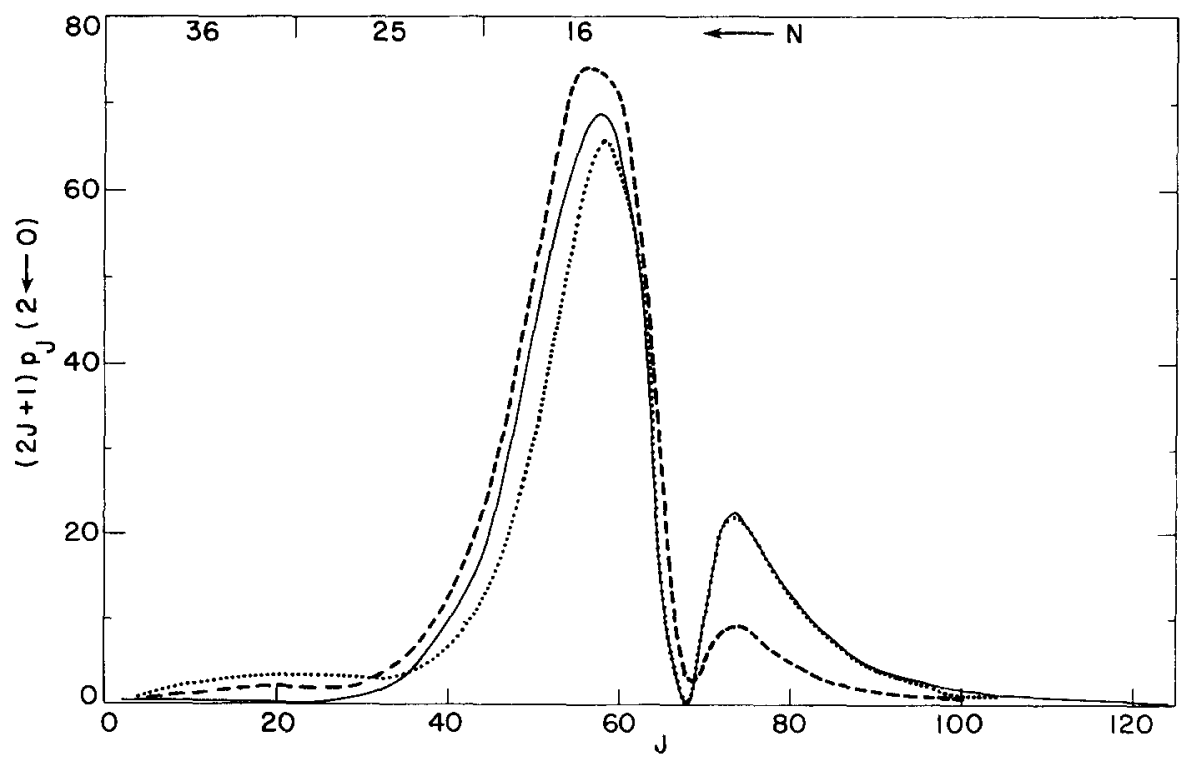

FIG. 5. Dimensionless partial cross sections $(2 J$ +1) $\mathcal{O}_{J}(2-0)$ for $\mathrm{Ar}+\mathrm{N}_{2}$. The notation is the same as in Fig. 3. 
TABLE ШI. Rotationally inelastic integral cross sections $\sigma\left(j_{f}-j_{i}\right)$ for Ar $+\mathrm{TlF}$ collisions at total energy $E /$ $k_{B}=1344^{\circ} \mathrm{K}$. The units are $\AA^{2}$. Also listed are the percent deviations from the accurate $\mathrm{CC}$ value. In the mixed methods the change from small $J$ method to large $J$ method was made at $J=210$.

\begin{tabular}{lcrcr}
\hline \multicolumn{1}{c}{ Method } & $\sigma(2 \leftarrow 0)$ & \multicolumn{1}{c}{$\%$} & $\sigma(4 \leftarrow 0)$ & \multicolumn{1}{c}{$\%$} \\
\hline CC & 58.9 & $\ldots$ & 16.4 & $\cdots$ \\
IOS & 69.6 & 18.4 & 29.1 & 77.4 \\
FOS & 69.3 & 17.9 & 28.3 & 72.6 \\
IOS + CC & 59.0 & 0.3 & 16.5 & 0.6 \\
FOS + CC & 59.0 & 0.3 & 15.5 & -5.5 \\
IOS + EBDW & 59.5 & 1.2 & 18.6 & 13.4 \\
FOS + EBDW & 59.5 & 1.2 & 17.5 & 6.7 \\
\hline
\end{tabular}

figure is listed the $N$ required for convergence. One sees that the IOS results are a very good approximation to the exact CC results for small $J$. As $J$ increases the IOS results slowly get out of phase and become, as one might expect, considerably too large in the large $J$, weak-coupling region. It may be noted that the minimum near $J=200$ is due to cancellation between the short and long range potentials. Scattering for $J>200$ is dominated by the long range tail of the potential, and scattering for $J<200$ is dominated by the short range repulsive potential. In the small $J$ region competition between many strongly coupled channels markedly damps the probability of a transition to any one channel.

In Fig. 3 the FOS results are compared with the IOS results for this same $0-2$ transition; they become identical to the IOS results at larger $J$. At small $J$ the FOS approximation gets out of phase but has about the right magnitude. Since the inte- gral inelastic cross section $\sigma(2-0)$ is proportional to the area under the curve, it is clear that the IOS and FOS cross sections are nearly equal. Also plotted for large $J$ on Fig. 3 are the CC results and the exponential Born (EBDW) results of Balint-Kurti and Levine. ${ }^{13}$ The EBDW approximation is better than the sudden approximations for large $J$ but is known to fail completely for small $J$ in this case. ${ }^{13}$

In Fig. 4 the converged FOS, IOS, EBDW, and $C C$ values of $(2 J+1) P_{J}$ are plotted for the $0 \rightarrow 4$ transition. The behavior is similar.

The availability of different simple approximations valid in different $J$ regions immediately suggests calculation of integral inelastic cross sections using one approximation for small $J$ and another for large $J$. Accordingly, in Table III we present the integral inelastic cross sections calculated in the $\mathrm{CC}$, IOS, FOS approximations and several combinations in which one approximation is used for $J<210$ and another for $J \geqslant 210$. Several of the simple methods are seen to give integral cross sections accurate to within a few percent.

It is also worth noting that because of the damping and associated small contribution of the dominant coupling (small $J$ ) region, the integral cross sections converge much more rapidly with $N$ than did the small $J$ opacity functions (partial cross sections). This is illustrated by Table IV, which gives the integral cross sections as functions of $N$.

$$
\text { B. } \mathrm{Ar}+\mathrm{N}_{2}
$$

Calculations of the $0 \rightarrow 2$ and $0 \rightarrow 4$ cross sections were also carried out for room temperature Ar$\mathrm{N}_{2}$ collisions using the potential parameters of

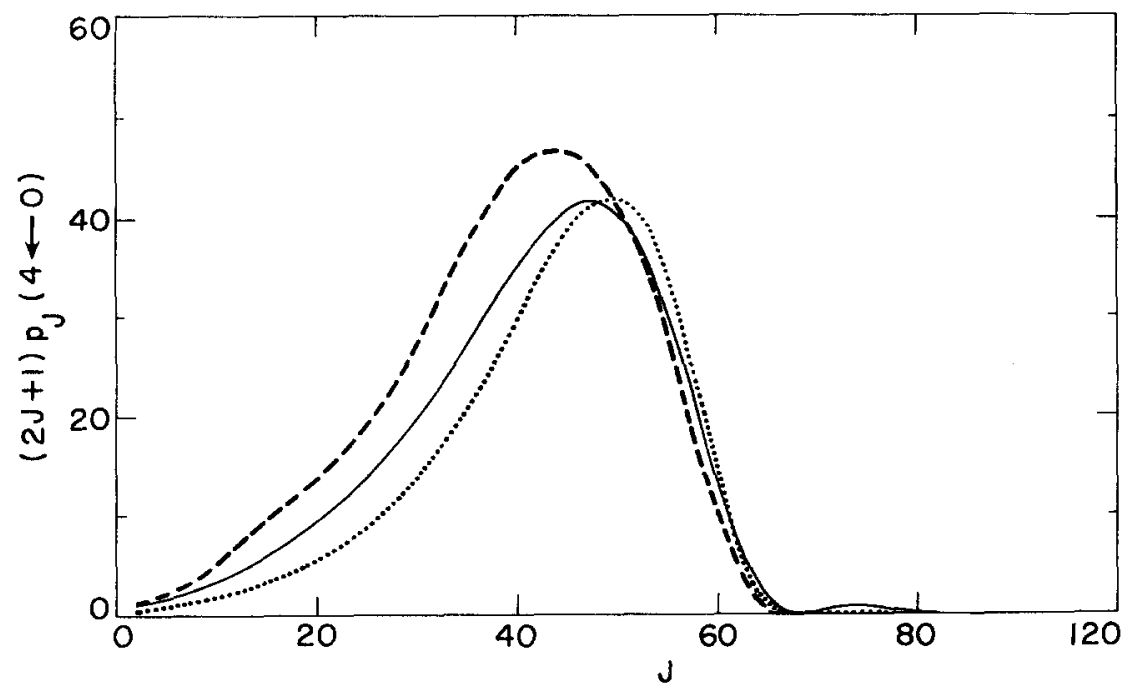

FIG. 6. Dimensionless partial cross sections $(2 J$ $+1) \sigma_{J}(4-0)$ for $\mathrm{Ar}+\mathrm{N}_{2}$. The notation is that of Fig. 3. 
TABLE IV. Convergence of integral inelastic cross sections $\sigma\left(j_{f}-j_{i}\right)$ for Ar $+\mathrm{TlF}$ as the number of channels $(N)$ is increased. The units are $\AA^{2}$.

\begin{tabular}{cccccccc}
\hline & & \multicolumn{7}{c}{$N$} \\
\cline { 3 - 8 } Cross section & Method & 9 & $\mathbf{1 6}$ & 25 & 36 & 49 & 64 \\
\hline \multirow{3}{*}{$\sigma(2-0)$} & CC & 65.2 & 62.8 & 57.3 & 58.8 & 58.9 & $\ldots$ \\
& IOS & 74.7 & 73.3 & 69.7 & 69.4 & 69.6 & 69.6 \\
& FOS & 76.6 & 73.7 & 68.7 & 69.9 & 69.3 & 69.4 \\
$\sigma(4-0)$ & CC & 33.7 & 24.1 & 20.2 & 16.3 & 16.4 & $\ldots$ \\
& IOS & 45.2 & 38.2 & 32.2 & 28.3 & 29.1 & 29.0 \\
& FOS & 43.0 & 39.5 & 29.7 & 28.6 & 28.3 & 28.2 \\
\hline
\end{tabular}

Pattengill, LaBudde, Bernstein, and Curtiss. ${ }^{16}$ The resulting IOS, FOS, and $\mathrm{CC}$ opacity functions are compared in Figs. 5 and 6 . From the number of channels required for convergence [listed at the top of Fig. 5] one sees that this system is not so strongly coupled as the previous one. However, the depression of the partial cross sections for $J \leq 40$ is a strong coupling effect.

Although the sudden approximations are not quite as well justified in this system as in the previous one, they still work quite well in the small $J(J$ $\leq 68$ here) region. It is interesting that for $J<35$, the FOS results for the $0 \rightarrow 2$ transition are better than those of the IOS approximation, which gives too much dominant coupling.

The large $J$ region where the sudden approximations are poor contributes only a very small fraction of the integral cross sections (given in Table $\mathrm{V})$ for this system, and nothing is gained by using one approximation for small $J$ and another for large $J$. However, the IOS and FOS integral cross sections are still accurate enough to be useful.

The existence and extent of the dominant coupling region in this problem depends very strongly on the repulsive asymmetry parameter $a_{12}$ whose value is still uncertain. It is interesting to note that if $a_{12}$ were decreased from 0.5 to an earlier estimate ${ }^{17}$ of 0.375 with the other parameters fixed, the dominant coupling region disappears, and no more than 16 channels are required for convergence at any $J$. This is illustrated in Fig. 7, where the FOS values of $(2 J+1) \mathcal{P}_{J}(2,0)$ obtained with the two values of $a_{12}$ are compared.

$$
\text { C. } \mathrm{Ar}+\mathrm{F}_{2}, \mathrm{Cl}_{2}, \mathrm{Br}_{2} \text {, and } \mathrm{I}_{2}
$$

To check further the observation that in the two previous examples the well-known FOS approximation gives reasonable results for small $J$, even in dominant coupling regions, and to see how the

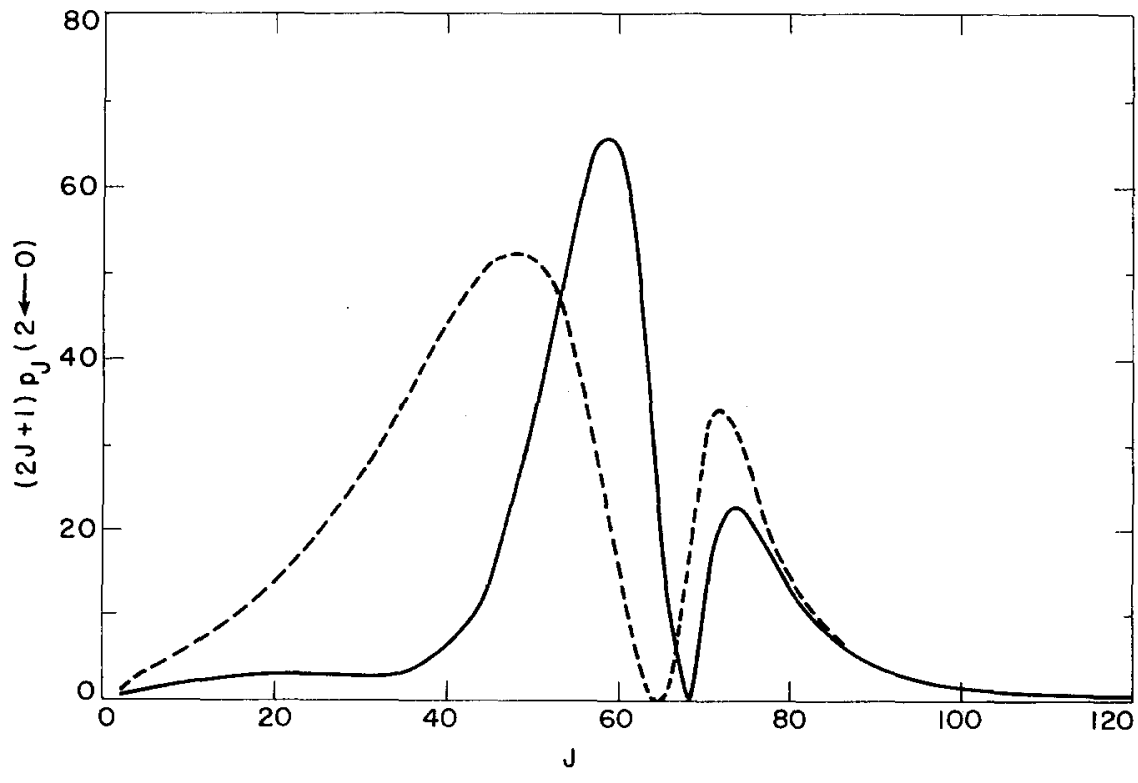

FIG. 7. Effect of the size of the repulsive asymmetry parameter $a_{12}$ on the FOS dimensionless partial cross sections $(2 J+1) \rho_{J}$ $(2-0)$ for $\mathrm{Ar}+\mathrm{N}_{2}$. The solid line is with $a_{12}=0.5$; the dotted line is with $a_{12}$ $=0.375$. 
TABLE V. Rotationally inelastic integral cross sections $\sigma\left(j_{f}-j_{i}\right)$ for $\mathrm{Ar}+\mathrm{N}_{2}$ collisions at total energy $E / k_{B}$ $=300^{\circ} \mathrm{K}$. The units are $\AA^{2}$. Also listed are the percent deviations from the accurate $\mathrm{CC}$ value.

\begin{tabular}{lcccc}
\hline \hline Method & $\sigma(2-0)$ & $\%$ & $\sigma(4-0)$ & $\%$ \\
\hline CC & 22.5 & $\ldots$ & 22.4 & $\cdots$ \\
IOS & 22.3 & -0.9 & 18.8 & -16 \\
FOS & 21.1 & -6.2 & 16.7 & -25 \\
\hline \hline
\end{tabular}

number $N$ of coupled channels required increases with increasing reduced mass, we calculated the FOS and IOS values of some inelastic cross sections on a series of model problems intended to represent scattering of $\mathrm{Ar}$ atoms by $\mathrm{F}_{2}, \mathrm{Cl}_{2}, \mathrm{Br}_{2}$, and $\mathrm{I}_{2}$, respectively. The parameters of the spherical part of each intermolecular potential (see Table I) were obtained using the parameters and combining rules of Hirschfelder, Curtiss, and Bird $^{18}$ and should be fairly reasonable estimates; however, the asymmetry parameters were arbitrarily kept fixed at 0.20 .

The resulting integral inelastic cross sections (in units of $\AA^{2}$ ) are presented in Table VI along with the maximum number of channels required to obtain convergence of all partial cross sections to within 5\%. Again, the FOS results are good approximations to the IOS results regardless of the number of channels involved.

\section{DISCUSSION AND CONCLUSIONS}

From the results of this paper, we conclude that the rotationally inelastic scattering problem is much less difficult than the large number of coupled rotational states would make it appear to be. This is due to the fact that the region where large numbers of states are strongly coupled and competing makes a small contribution to the inelastic cross sections and can hence be safely treated with rather simple approximations. In particular, the IOS and the well-known FOS approximation provide computationally easy ways of obtaining rather good small $J$ partial cross sections for rotationally inelastic collisions of heavy diatomic molecules. For systems in which the large $J$ (weak-coupling long-range) region gives a large contribution to the integral cross section, one can calculate reliable cross sections economically using a sudden approximation at small $J$ and a $\mathrm{CC}$ or $\mathrm{EBDW}$ method in the large $J$ region where only a few channels are needed.

The major reason for our present interest in the FOS approximation, since the IOS method is already computationally very simple, is that the FOS method is easily applied to potentials more
TABLE VI. Integral inelastic cross sections $\sigma\left(j_{f}-j_{i}\right)$ (in $\breve{A}^{2}$ ) for room temperature collisions of Ar with $F_{2}$. $\mathrm{Cl}_{2}, \mathrm{Br}_{2}$, and $\mathrm{I}_{2}$.

\begin{tabular}{lccrr}
\hline System & Approx. & $N$ & $\sigma(2-0)$ & $\sigma(4-0)$ \\
\hline $\mathrm{Ar}+\mathrm{F}_{2}$ & FOS & 25 & 55.72 & 17.23 \\
$\mathrm{Ar}+\mathrm{F}_{2}$ & IOS & & 51.29 & 17.03 \\
$\mathrm{Ar}+\mathrm{Cl}_{2}$ & FOS & 36 & 55.15 & 36.38 \\
$\mathrm{Ar}+\mathrm{Cl}_{2}$ & IOS & & 55.72 & 36.39 \\
$\mathrm{Ar}+\mathrm{Br}_{2}$ & FOS & \multirow{2}{*}{36} & 44.38 & 35.26 \\
$\mathrm{Ar}+\mathrm{Br}_{2}$ & IOS & & 46.40 & 34.15 \\
$\mathrm{Ar}+\mathrm{I}_{2}$ & FOS & \multirow{2}{*}{64} & 51.91 & 34.14 \\
$\mathrm{Ar}+\mathrm{I}_{2}$ & IOS & & 51.44 & 31.93 \\
\hline
\end{tabular}

general than that used here. For example, the FOS approximation can be applied to potentials of the form

$$
U\left(r^{\prime}, \theta\right)=\sum_{n=0}^{\infty} U_{n}\left(r^{\prime}\right) P_{n}(\cos \theta)
$$

by direct generalization of Eqs. (15) and (17). As formulated herein, the IOS approximation cannot be used for such a potential unless $U_{n}\left(r^{\prime}\right) \propto U_{m}\left(r^{\prime}\right)$ for all $n, m>0$. However, as this work was being completed, it was discovered ${ }^{19}$ that by using bodyfixed coordinate axes a drastic simplification can always be achieved via an IOS approximation, regardless of the form of the potential. Furthermore, this approach will also produce a marked increase in the computation speed of both the sudden approximations. Research along this line will be reported in a subsequent paper. ${ }^{19}$

\section{ACKNOWLEDGMENT}

We thank the Department of Chemistry of Brigham Young University for the generous share of its computing budget which made the $\mathrm{CC}$ computations possible.

\footnotetext{
* Research supported in part by grants from Brigham Young University.

${ }^{*}$ Present address: Department of Chemistry, McGill University, Montreal 110, Quebec, Canada.

${ }^{\ddagger}$ Present (temporary) address: $T-6$, University of California-Los Alamos Scientific Laboratory, Los Alamos, NM 87544.

${ }^{2}$ R. G. Gordon, J. Chem. Phys. 51, 14 (1969).

${ }^{2}$ W. N. Sams and D. J. Kouri, J. Chem. Phys. 51, 4809 (1969); J. Chem. Phys. 51, 4815 (1969); J. Chem. Phys. 52, 4144 (1970); and J. Chem. Phys. 53, 496 (1970).

${ }^{3}$ R. B. Bernstein, A. Dalgarno, H. S. W. Massey, and I. C. Percival, Proc. Roy. Soc. (London) A 274, 427 (1963).

${ }^{4}$ W. A. Lester, Jr. and R. B. Bernstein, J. Chem. Phys. 53, 11 (1970).

${ }^{5}$ A. M. Arthurs and A. Dalgarno, Proc. R. Soc. A 256, 540
} 
(1960)

${ }^{6} 1$. C. Percival and M. J. Seaton, Proc. Camb. Phil. Soc. 53, 654 (1957).

${ }^{7}$ R. D. Levine and R. B. Bernstein, J. Chem. Phys. 53, 686 (1970)

${ }^{8}$ R. G. Gordon, Program 187, Quantum Chemistry Program Exchange, Indiana University, 1969. We thank W. A. Lester, Jr. and D. G. Truhlar for a list of the errors in the QCPE version of this program.

${ }^{9}$ T. P. Tsien and R. T Pack, Chem. Phys. Lett. 6, 54 (1970); J. Chem. Phys. 6, 400 (1970); and J. Chem. Phys. 8, 579 (1971)

${ }^{11}$ R. T Pack, Chem. Phys. Lett. 14, 393 (1972).

"See, for example, R. J. Cross, Jr., J. Chem. Phys. 51, 5163

(1969); R. W. Fenstermaker, C. F. Curtiss, and R. B. Bernstein, J. Chem. Phys. 51, 2439 (1969).
R. D. Levine, Mol. Phys. 22, 497 (1971), and references therein. ${ }^{13} \mathrm{G}$. G. Balint-Kurti and R. D. Levine, Chem. Phys. Lett. 7, 107 (1970).

${ }^{14}$ R. T Pack and J. S. Dahler, J. Chem. Phys. 50, 2397 (1969); W. D. Smith and R. T Pack, J. Chem. Phys. 52, 1381 (1970).

${ }^{15} \mathrm{~A}$ more realistic Ar + TIF potential has been given by J. van de Ree and J. G. R. Okel, J. Chem. Phys. 54, 589 (1971)

${ }^{16}$ M. D. Pattengill, R. A. LaBudde, R. B. Bernstein, and C. F. Curtiss, J. Chem. Phys. 55, 5517 (1971).

${ }^{17}$ W. Erlewein, M. von Seggern, and J. P. Toennies, Z. Phys. 211, 35 (1968).

${ }^{18}$ J. O. Hirschfelder, C. F. Curtiss, and R. B. Bird, Molecular Theory of Gases and Liquids (Wiley, New York, 1964).

${ }^{19}$ R. T Pack, J. Chem. Phys. (to be published). 